Tohoku J. exp. Med., 1978, 124, 73-82

\title{
Diagnosis of Placental Function by Prediction Gurves for Heat-Stable Alkaline Phosphatase (HSAP)
}

\author{
Ryuji Yamaguchi and Naoyuki Shimozato \\ Department of Obstetrics and Gynecology, Nara Medical \\ University, Kashihara 634
}

\begin{abstract}
Yamaguchn, R. and Shrmozato, N. Diagnosis of Placental Function by Prediction Curves for Heat-Stable Alkaline Phosphatase (HSAP). Tohoku J. exp. Med., 1978, 124 (1), 73-82 — In order to establish an effective diagnostic method for placental function using HSAP, the authors formulated two expressions to predict HSAP values for their rise before 38.7 weeks of gestation and declining after that; $\log _{e} y=1.03 a-3.37+0.123 x$ (i), and $y=Y(1+d / 100)$ (ii), respectively. Further they prepared a chart of curves for these predicting expressions. The results obtained are as follows: (1) In normal pregnancies the deviation of prediction from measured values of HSAP was $\pm 15 \%$ in $94 \%$ of the cases examined. (2) Many of cases of abnormal pregnancy showed a low level of HSAP, $-15 \%$ or lower, and abnormal signs in HSAP pattern were found several days or weeks prior to the onset of fetal troubles. (3) Prolonged pregnancy with fetal troubles was able to be distinguished from "false" prolonged pregnancy. (4) In two consecutive pregnancies in the same woman, the courses of HSAP were strikingly consistent as far as both pregnancies were normal. The last fact suggests that the placental production of HSAP and its release into the maternal blood may be influenced by the individual constant $a$ in expression (i). (5) It is recommended that the HSAP prediction curve is worthy to use as an easy screening method for placental function in the third trimester of pregnancy.
\end{abstract}

Heat-stable alkaline phosphatase (HSAP) in maternal serum, since Neale and his associates (McMaster et al. 1964; Neale et al. 1965) demonstrated its heatstability and origin in the placenta, has been used by many researchers in an attempt to utilize its placenta-specificity as a placental function test in late pregnancy. However, despite its convenience in sampling, measurement and material's stability, HSAP levels in normal pregnancy are usually too widely distributed to define a normal range. All efforts so far have failed in establishing any criteria for interpreting placental function with HSAP levels.

In our previous report (Yamaguchi et al. 1968), on the basis of the repeated measurement of HSAP in the same women, we suggested that the pattern of HSAP is more reliable for diagnosis of placental function than the change in level itself. More recently, we have found that the rate of elevation of HSAP with the

Received for publication, June 17, 1977.

Supported by Grant for Researches on Handicapped Children 1976, from the Ministry of Health and Welfare, Japan.

Reprint requests: Dr. Ryuji YaMaGUCHI, Department of Obstetrics and Gynecology, Nara Medical University, Kashihara, Nara 634, Japan. 
progress of pregnancy is almost constant independent of the height in its individual level (Yamaguchi et al. 1975). Based on these findings, we formulated expressions for prediction of HSAP levels and further obtained a chart of curves for these changes to interpret the behavior of HSAP. Our procedures and results are summarized in this report.

\section{Materials and Methods}

Materials. Data used were from 328 normal and 68 abnormal pregnancies seen in Nara Medical University Hospital during a three-year period of 1973-1975. These included the data of 35 cases in their repeated pregnancies.

$M e a s u r e m e n t$ of $H S A P$. In these pregnant women, serum was heat-treated at $65^{\circ} \mathrm{C}$ for $5 \mathrm{~min}$, and HSAP was determined using a commercial alkaline phosphatase kit. The values of enzyme activity was expressed in King-Armstrong unit.

Whether pregnancy is normal or not was finally judged on the feature and data of the clinical course of pregnancy and the fetal conditions in pregnancy and at delivery, and also with reference to the results of other tests for placental function.

\section{RESULTS}

Expressions for prediction of HSAP values

In the previous report (Yamaguchi et al. 1975), we suggested that in normal pregnancy, HSAP, independent of its individual variations in serum level, was detected in the maternal serum at a certain fixed elevation rate (about $2-3 \%$ per day) until the 38th or the 39th week of gestation. On the hypothesis that despite individual variations in height, the elevation rate of HSAP is individually constant, we tried to introduce the expressions to predict HSAP values in the

TABLE 1. Maternal serum heat-stable alkaline phosphatase in 170 normal pregnancies ( $K-A$ units)

\begin{tabular}{cccr}
\hline $\begin{array}{c}\text { Weeks of } \\
\text { pregnancy }\end{array}$ & $\begin{array}{c}\text { Number of } \\
\text { determinations }\end{array}$ & $\begin{array}{c}\text { Mean } \pm \text { s.D. } \\
\text { (K-A units) }\end{array}$ & Upper-Lower \\
\hline 27 & 12 & $2.4 \pm 0.8$ & $4.1-1.4$ \\
28 & 13 & $3.4 \pm 0.9$ & $5.8-2.4$ \\
29 & 12 & $3.5 \pm 2.2$ & $7.5-1.0$ \\
30 & 16 & $4.7 \pm 3.2$ & $12.0-1.0$ \\
31 & 21 & $4.7 \pm 1.6$ & $7.5-1.0$ \\
32 & 24 & $5.5 \pm 3.1$ & $13.0-1.0$ \\
33 & 43 & $6.7 \pm 3.2$ & $17.8-1.0$ \\
34 & 44 & $7.0 \pm 4.1$ & $25.4-2.1$ \\
35 & 53 & $8.3 \pm 4.1$ & $29.2-2.7$ \\
36 & 70 & $9.1 \pm 4.6$ & $30.2-2.7$ \\
37 & 88 & $10.1 \pm 4.5$ & $29.5-3.1$ \\
38 & 102 & $11.7 \pm 4.1$ & $24.0-3.8$ \\
39 & 92 & $12.9 \pm 5.4$ & $30.5-3.4$ \\
40 & 100 & $13.8 \pm 6.2$ & $37.7-4.1$ \\
41 & 47 & $14.0 \pm 6.6$ & $42.5-4.8$ \\
$42^{*}$ & 15 & $13.8 \pm 3.4$ & $17.8-8.7$ \\
$43^{*}$ & 7 & $7.7 \pm 5.0$ & $18.9-5.7$ \\
$44^{*}$ & 2 & 6.8 & $9.6-3.9$ \\
\hline
\end{tabular}

* Prolonged pregnancy. 


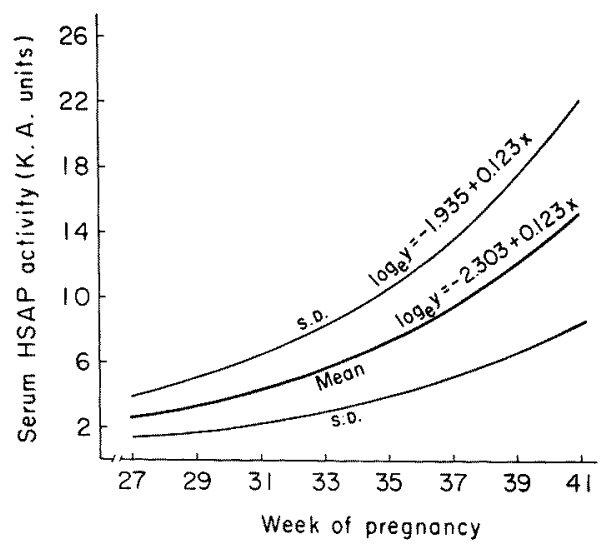

Fig. 1. Regression curves for the mean and standard deviation of HSAP values in 170 cases of normal pregnancy.

third trimester using 752 serum specimens from 170 cases of normal pregnancy. HSAP values in each pregnancy week are shown in Table 1 . From that, two regression lines for elevation of HSAP are obtained; one is for the mean, and the other for the standard deviation (Fig. 1). The general expression to optional HSAP value $y$ within the mean and the standard deviation is able to be introduced by using the variable $a$ which proportionally varies according to the difference between $y$ from the mean value. That is,

$$
\log _{e} y=1.03 a-3.37+0.123 x
$$

In expression (1), $x$ shows weeks of gestation in term of decimal notation; $y$ is the value of HSAP in K-A unit; $a$ is the constant for an individual pregnant woman decided by the value of HSAP $y$ in the first measurement at some week arbitrarily taken in the course of gestation around 30-32 weeks.

Elevation rate of HSAP, however, becomes slack after around 38-39 weeks as shown in Table 1. When the slacking rate was calculated using normal 38-42 weeks cases, the rate approximately coincided with a parabolic curve to the elevation curve introduced from expression (1) (Fig. 2). Thus, combining expression (1) with the slacking rate, the following two expressions were obtained:

$$
\begin{aligned}
& y=Y(1+d / 100) \\
& d=-2\left(x^{\prime}-38.7\right)^{2}
\end{aligned}
$$

In expression (2) and $\left(2^{\prime}\right) d$ shows the slacking rate of HSAP; $y$ indicates the predicted value of elevated HSAP after 38.7 weeks that can be obtained with expression (1); $x^{\prime}$ shows the weeks of gestation expressed in decimal notation exclusively applicable for 38.7 weeks and later.

The value of 38.7 in expression $\left(2^{\prime}\right)$ is obtained as follows: When expression $\left(2^{\prime}\right)$ is expressed as $d=-2\left(x^{\prime}-\mathrm{t}\right)^{2}$, the maximum $y$ in expression (2) is to be gotten when $d$ is zero. On the other hand, from the data in Table 1, the maximum value of 


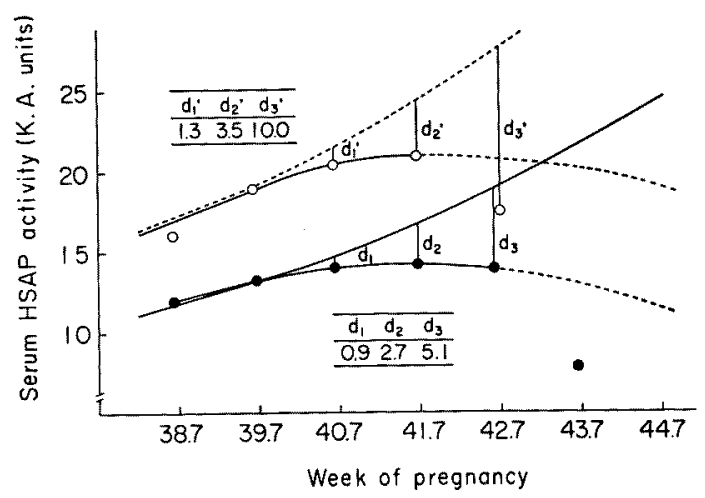

Fig. 2. Slacking curves for the mean and standard deviation after 38.7 weeks in normal pregnancy.

- (predicted value for elevation of mean) - (actual mean value). o-o (predicted value for elevation of S.D.) - (actual s.D. value).

HSAP is assumed to exist at 41.4 weeks (middle day of 7 days in 41 weeks). The predicted value of HSAP at 41.4 weeks is calculated as $Y=16.268$ from expression (1). Next, by substitution of this 16.268 into $y=Y\left(1-2(x-t)^{2} / 100\right)$, which is the general form of expression (2), $14.0=16.268(1-2(41.4-t) / 100)^{2}$, then the value of $t$ can be calculated as $38.7597(\fallingdotseq 38.7)$. Thus, obtained $t$ indicates the contact point (weeks) of two curves derived from expressions (1) and (2). Therefore, expression (1) can be used up to about 39 weeks, or actually up to 38.7 weeks in full; on the contrary, expression (2) is to be applied after 38.7 weeks and later.

Example of actual calculation for expression (1): In a woman of full 30 weeks and 2 days of gestation, the weeks are shown by 30.3 weeks because 2 days is $2 / 7 \fallingdotseq 0.3$. When HSAP $y$ on that day shows $4.7 \mathrm{~K}-\mathrm{A}$ units, this woman's constant $a$ will be 1.1559825 . With this value, her HSAP level at 38 weeks and 2 days $(x \div 38.3)$ can be estimated as

$$
\log _{\iota} y=-2.1793381+0.123 \times 38.3=2.5315629
$$

equivalent to, $y=12.57313 \fallingdotseq 12.5$ (K-A units).

Prediction chart for HSAP and its use

To avoid troublesome calculation in each case, we prepared a chart for predicted values of HSAP using the results of preparatory calculation for estimation (Fig. 3). In this chart, some examples of normal pregnancy with their predicted and actually measured values of HSAP were plotted. The figure clearly tells that, except slight decline of HSAP several days prior to confinement in some cases, predicted and measured values strikingly agree to each other. From the preconfinement decline of HSAP, as far as prengnacy is normal, it is often possible to predict the approach of parturition. 


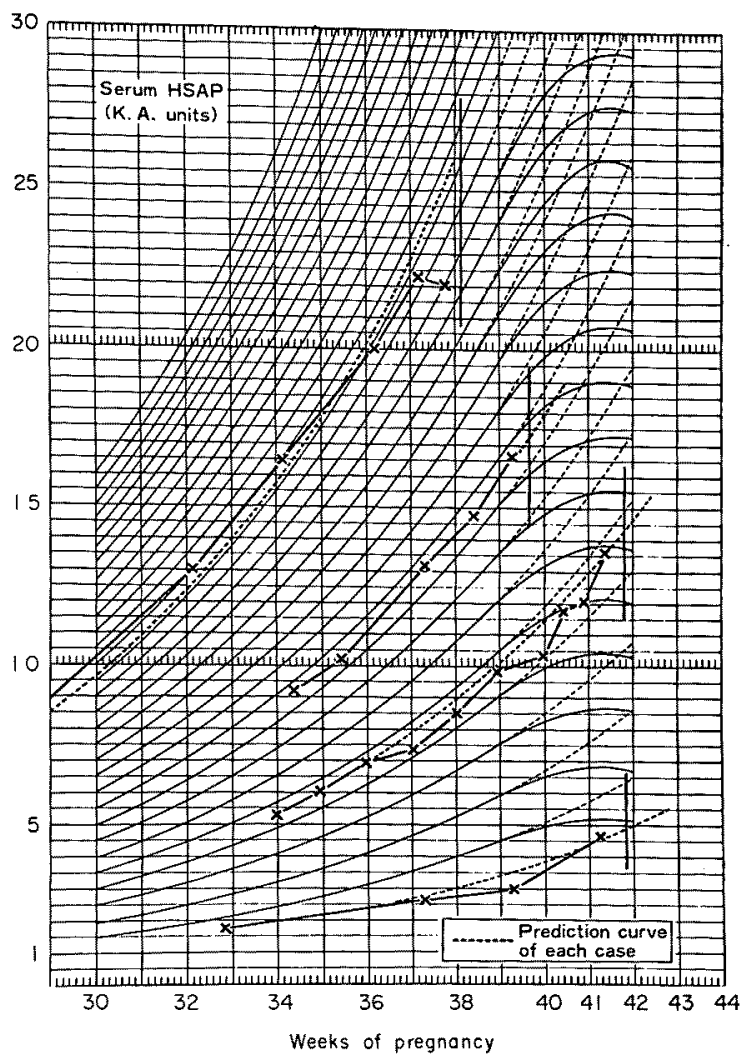

Fig. 3. Prediction chart of HSAP and courses of actually measured HSAP in several cases of normal pregnancy.

\section{Deviation of predicted from measured values of HSAP}

In order to confirm the general applicability of our method, examination was made in other 150 women of normal pregnancy. Among the values of HSAP measured twice or more in each case, one showing the greatest difference from the predicted value was taken up, and expressed by $(+\%)$ for the figure higher and by $(-\%)$ for that lower than the predicted value. As shown in Fig. 4 , the differences between measured and predicted values almost stood within $\pm 15 \%$ in $94 \%$ of all cases showing a normal distribution.

\section{Behavior of HSAP in abnormal pregnancy}

In various abnormal pregnancies, how predicted and actually measured HSAP values vary was studied in some typical examples (Fig. 5). In the light of the prediction curves, it is possible to elucidate whether any elevation of HSAP levels is apparent or real. Also, in cases of severe toxemia or prolonged pregnancy with fetal troubles, it can be re-affirmed on the curves that they take an "unstable" pattern as we have previously reported (Yamaguchi et al. 1968). 


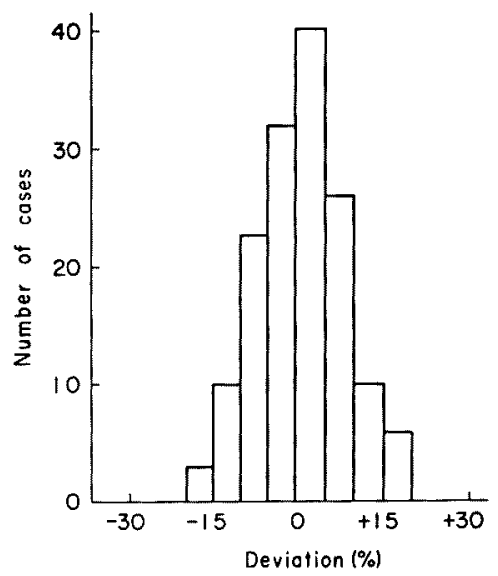

Fig. 4. Distribution of deviation percentages between predicted and measured HSAP values in other 150 cases of normal pregnancy.

$$
\text { Deviation }(\%)=\frac{\text { predicted }- \text { measured }}{\text { predicted }} \times 100
$$

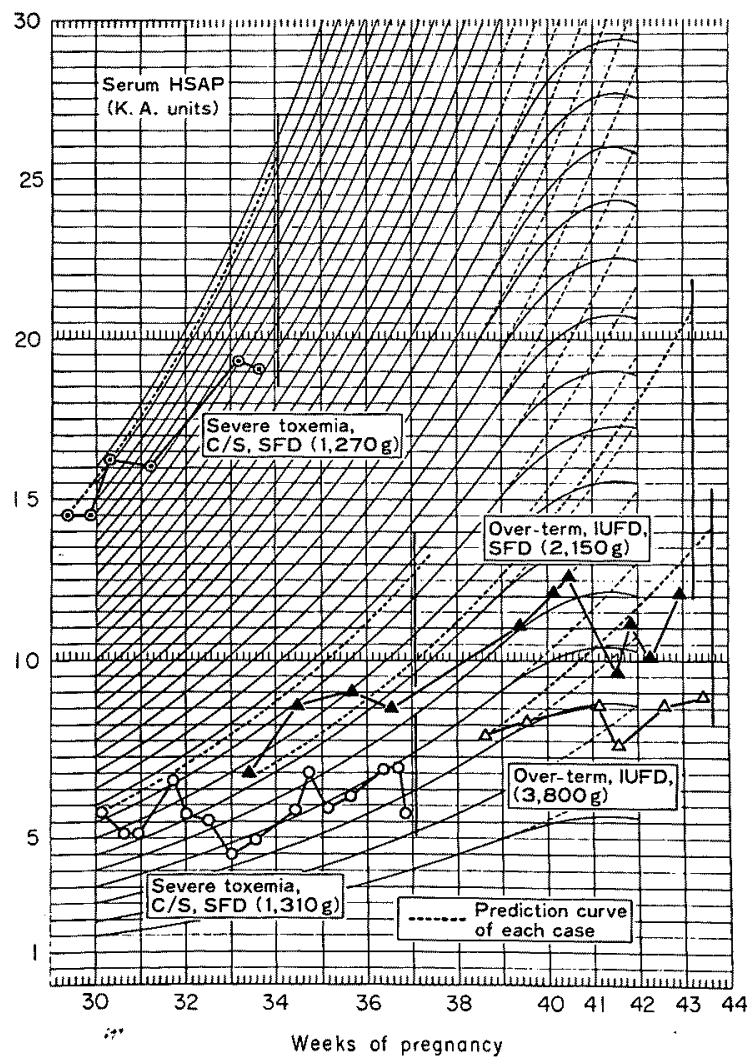

Fig. 5. HSAP courses in several cases of abnormal pregnancies plotted in the prediction chart. 


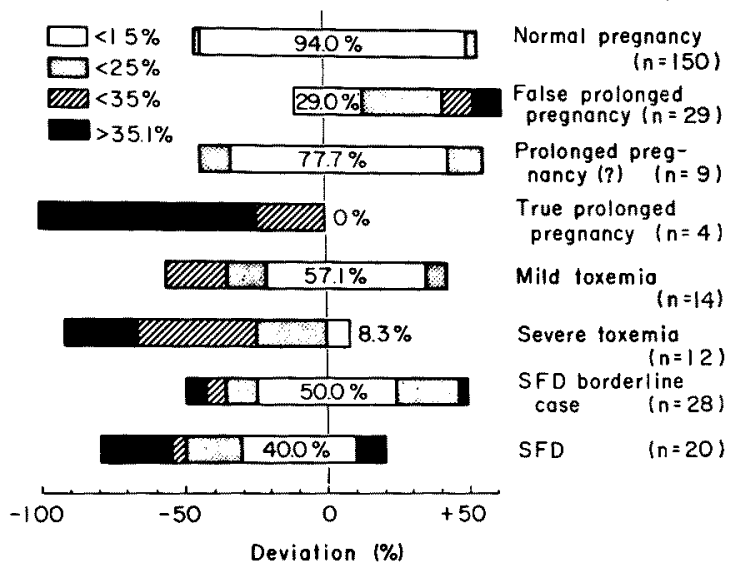

Fig. 6. Deviation features of HSAP from predicted values in normal and abnormal pregnancies (distribution percentage in each pregnancy group).

TABLE 2. Fetal body weight, Apgar score and placental weight at birth in each pregnancy group shown in Fig. 6

\begin{tabular}{|c|c|c|c|}
\hline & $\begin{array}{l}\text { Body weight at birth } \\
(\text { Mean } \pm \text { S.D. })\end{array}$ & $\begin{array}{c}\text { Apgar score (1 min) } \\
(\text { Mean } \pm \text { s.d. })\end{array}$ & $\begin{array}{l}\text { Placental weight } \\
\text { (Mean } \pm \text { S.D.) }\end{array}$ \\
\hline Normal $(n=150)$ & $3218.3 \pm 408.60$ & $8.9 \pm 0.69$ & $579.2 \pm 102.09$ \\
\hline \multicolumn{4}{|l|}{ Prolonged pregnancy } \\
\hline False $(n=29)$ & $3407.9 \pm 455.70$ & $8.9 \pm 0.44$ & $631.4 \pm 124.12$ \\
\hline $\begin{array}{l}\text { Without fetal troubles } \\
\qquad(n=9)\end{array}$ & $3243.3 \pm 425.10$ & $6.3 \pm 3.16$ & $608.3 \pm 95.85$ \\
\hline $\begin{array}{l}\text { With fetal troubles } \\
\qquad(n=4)\end{array}$ & $2907.5 \pm 795.75$ & $2.3 \pm 2.87$ & $487.5 \pm 102.43$ \\
\hline \multicolumn{4}{|l|}{ Toxemia } \\
\hline Mild $(n=14)$ & $2957.9 \pm 573.53$ & $8.4 \pm 1.02$ & $567.1 \pm 112.48$ \\
\hline Severe $(n=12)$ & $1881.7 \pm 776.84$ & $5.8 \pm 3.35$ & $423.8 \pm 217.83$ \\
\hline \multicolumn{4}{|l|}{ SFD } \\
\hline Borderline case $(n=28)$ & $2667.7 \pm 115.32$ & $8.9 \pm 0.94$ & $502.3 \pm 66.38$ \\
\hline True $(n=20)$ & $2191.8 \pm 267.15$ & $7.9 \pm 2.50$ & $439.5 \pm 104.85$ \\
\hline
\end{tabular}

Severity of toxemia was defined by the Committee of Toxemia of Japan Society of Obstetrics and Gymecology (1962).

Criteria of SFD was defined by Funakawa (1968).

Fig. 6 shows the distribution percentages of deviation of predicted from measured values of HSAP in cases of normal and abnormal pregnancy. In both cases of prolonged pregnancy with fetal troubles and severe toxemia, the deviation was almost concentrated on the left minus side, while the cases clinically considered as "false" prolonged pregnancy from their menstrual irregularities had their deviation distributed more frequently on the right plus side. With toxemia of pregnancy, mild cases had the distribution on the minus side less frequently than severe cases.

In cases of SFD of unknown origin, without any maternal disease such as 
toxemia, the distribution of deviation percentages was inconsistent either coming on the minus side or remaining within a normal range. It is of interest that in a few cases of SFD the deviation was distributed on the plus side. This suggests that there exist some causes of SFD other than the placental "dysfunction" as expressed by HSAP.

\section{Behavior of HSAP in repeated pregnancy in the same individual}

How HSAP behaves in repeated pregnancies in the same woman was studied in 35 cases. The variation in HSAP behavior between two pregnancies in the same woman can be shown by the difference between two values of constant $a$ indicated in expression (1)-between the value in the first pregnancy $a_{1}$ and that in the second pregnancy $a_{2}$. The smaller the $a_{1}-a_{2}$, the closer to each other is the behavior of HSAP. As shown in Table 3, in 14 cases normal in both pregnancies (group A), the mean of $a_{1}-a_{2}$ was only 0.08 , while in 15 cases abnormal in either one of the two pregnancies (group B), the mean was 0.37 , and in the other 6 cases abnormal in both pregnancies (group $\mathrm{C}$ ), the mean was 0.40 . The differences of means between groups $\mathrm{B}$ and $\mathrm{C}$ and group $\mathrm{A}$ were significant at $p<0.05$. This may well confirm our hypothesis that the behavior of HSAP is influenced by the constant $a$ inherent in each pregnant woman as far as pregnancy (placenta) is normal, and further may suggest that the constant $a$ becomes impaired in abnormal pregnancy, disturbing the HSAP values.

TABLE 3. Values of $a_{1}-a_{2}$ in consecutive two pregnancies in same individuals

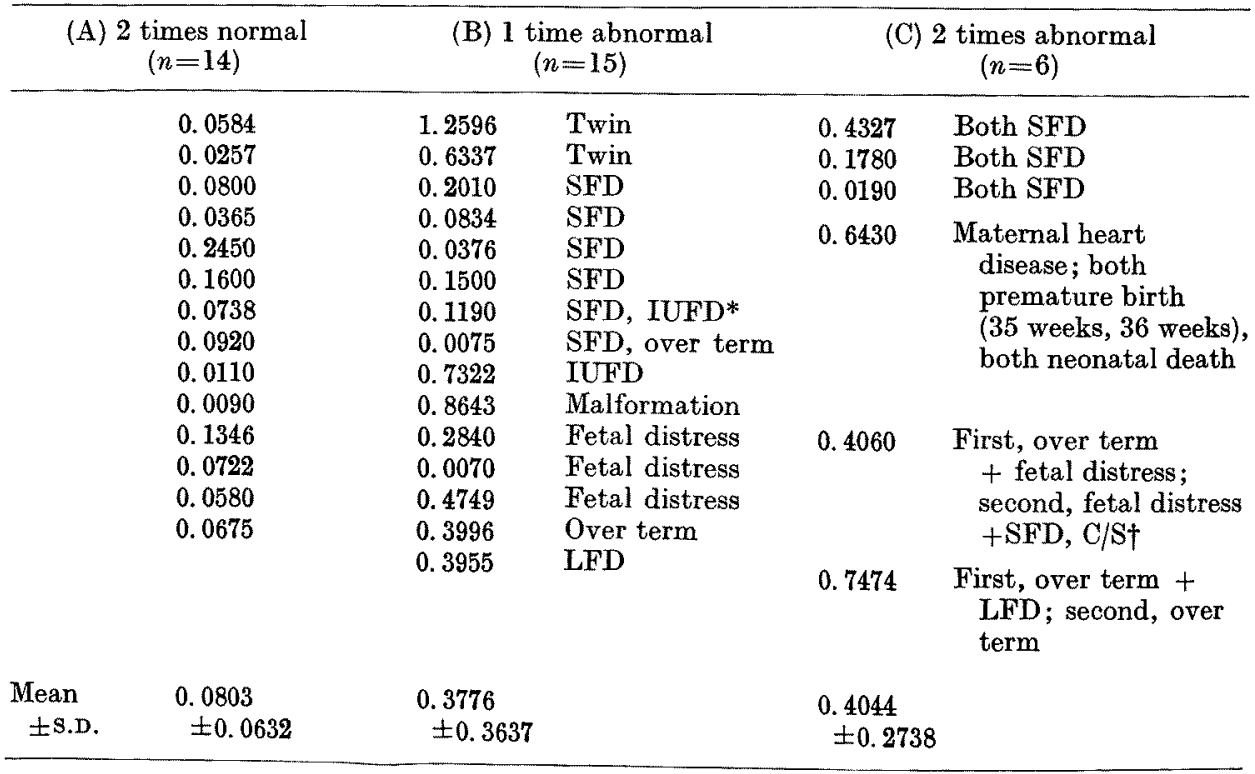

* IUFD: Intrauterine fetal death. †C/S: Cesarean section. 


\section{Drscussion}

HSAP, which is one of the most specific enzymes of the placenta, is highly attractive as a clinical index available for evaluation of placental function in late pregnancy, because of its easiness in sampling and determination. However, as described already, normal range of HSAP usually distributes too wide, eventually impeding the diagnostic value of HSAP. This tendency is common to other placenta-originating enzymes and substances inclusive of HSAP. In the present study, paving the way for reliable interpretation of HSAP behavior, it has been found that the elevation in HSAP level with the progress of gestation is definitely regulated by the constant $a$ individually inherent in each pregnancy or placenta. What is the most startling is the finding that the constant $a$ was almost identical in the first and the second pregnancies in the same woman as far as their pregnancies were normal. This suggests that the production of HSAP in the placenta and its release into the maternal blood are under the control of a certain schedule originally established in the placenta.

Our expressions for prediction of HSAP values presented in this report show that the formerly conceived HSAP pattern with so wide variations merely reflects a seeming phenomenon. In fact, the fluctuation in HSAP values is rather small compared with other tests for placental function.

With the use of our expressions for prediction of HSAP values and the chart of their curves, signs of placental dysfunction likely to affect the fetus can easily be screened, especially in cases of prolonged pregnancy which is one of the most crucial problems to obstetricians. It is interesting that abnormal HSAP patterns on the prediction chart sometimes appear several days or weeks prior to abnormal urinary estriol. This fact indicates that placental dysfunction occurs preceding a fetal deprivation by several days or weeks. Another finding of interest is that abnormal HSAP pattern does not always occur in all SFD with no maternal disease. But, this is not a matter of surprise when SFD is considered as a kind of syndrome. The fact further tends to suggest a limit of diagnosing all of placental function by HSAP alone.

In the same way as in HSAP, expressions for predicted values can be prepared for leucine aminopeptidase (LAP) and cystine aminopeptidase (CAP), although they are not always so reliable as HSAP. Anyway, our new method to analyse HSAP pattern, simple and easy, is considered worthy as a screening method to diagnose placental function in the third trimester of pregnancy.

\section{References}

1) Committee of Toxemia of Japan Society of Obstetrics and Gynecology (1962) Classification of pregnancy toxemia. J. Japan. Obstet. Gynec. Soc., 14, 1129-1130. (Japanese)

2) Funakawa, H. (1968) Gestational age and fetal growth. Acta neonat. jap., 4, 129133. (Japanese)

3) McMaster, Y., Tennant, R., Clubb, J.S. \& Neale, F.C. (1964) The mechanism of elevation of serum alkaline phosphatase in pregnancy. J. Obstet. Gynaec. Brit. Cwlth, 71, 735-739. 
4) Neale, F.C., Clubb, J.S., Hotchkiss, D. \& Posen, S. (1965) Heat-stability of human placental alkaline phosphatase. J. clin. Path., 18, 359-363.

5) Yamaguchi, R., Yoshida, T., Kimura, C. \& Hatakeyama, Y. (1968) Serum heatstable alkaline phosphatase and placental function. Tohoku J. exp. Med., 96, 327-331.

6) Yamaguchi, R., Nishikawa, Y., Itahashi, T., Nakano, Y., Shimozato, N., Takei, H., Matsuda, T. \& Ushioda, E. (1975) Placental function and its influence on the fetus. Obstet. Gynec. Therapy, 31, 117-123. (Japanese) 